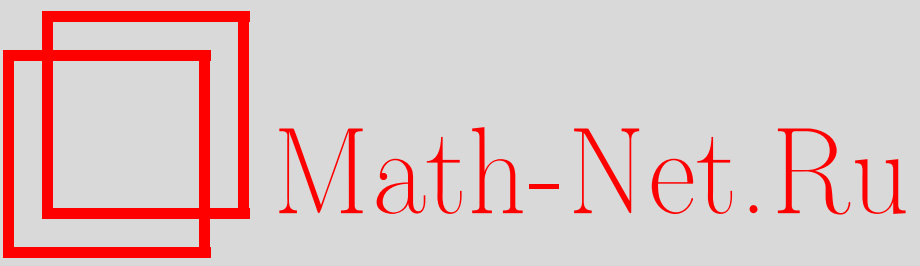

Т. Ф. Долгих, Методы решения задачи зонального электрофореза с периодическими начальными данными, Итоги науки и техн. Сер. Соврем. мат. и ее прил. Темат. обз., 2019, том 172, 38-47

DOI: https://doi.org/10.36535/0233-6723-2019-172-38-47

Использование Общероссийского математического портала Math-Net.Ru подразумевает, что вы прочитали и согласны с пользовательским соглашением

http://www.mathnet.ru/rus/agreement

Параметры загрузки:

IP : 35.173 .219 .12

26 апреля 2023 г., 14:39:56 
УДК 517.956, 517.958:531.32, 519.63

\title{
МЕТОДЫ РЕШЕНИЯ ЗАДАЧИ ЗОНАЛЬНОГО ЭЛЕКТРОФОРЕЗА С ПЕРИОДИЧЕСКИМИ НАЧАЛЬНЫМИ ДАННЫМИ
}

\author{
(c) 2019 г. $\quad$ T. $\Phi$. ДОЛГИХ
}

\begin{abstract}
АннотАция. Рассмотрена задача зонального электрофореза двухкомпонентной смеси с пространственно-периодическим начальным распределением компонент смеси. Предложены два способа решения: аналитический (метод годографа) и численный (метод конечных объемов). Проведен сравнительный анализ полученных результатов.
\end{abstract}

Ключевые слова: модель зонального электрофореза, метод годографа, метод конечных объемов, пространственно-периодические начальные данные.

\section{METHODS FOR SOLVING THE PROBLEM OF ZONAL ELECTROPHORESIS WITH PERIODIC INITIAL DATA}

\author{
(c) 2019 T. F. DOLGIKH
}

\begin{abstract}
We consider the problem of zonal electrophoresis of a two-component mixture with spatially periodic initial distribution of the mixture components. Two methods of solution are proposed: analytical (hodograph method) and numerical (method of finite volumes). A comparative analysis of the results obtained is performed.
\end{abstract}

Keywords and phrases: model of zone electrophoresis, hodograph method, method of finite volumes, spatially periodic initial data.

AMS Subject Classification: 35Q35, 35L03, 35J62

1. Введение. Процесс переноса и разделения смеси веществ на отдельные составляющие под действием электрического поля в достаточно длинном капилляре с непроницаемыми боковыми стенками называется зональным электрофорезом. Этот метод разделения широко применяется в медицине, биологии, химии, например, для определения и выделения составляющих многокомпонентной смеси.

В работе рассматривается начальная стадия зонального электрофореза, когда электромиграционные эффекты играют более значимую роль в изменении профиля концентраций индивидуальных компонент смеси, чем диффузионные эффекты, что позволяет исследовать бездиффузионную модель в случае сильных электролитов, когда вещества полностью диссоциировали на ионы, и нейтральные элементы кроме растворителя (воды) в смеси отсутствуют.

В зависимости от параметров компонент (например, зарядности, подвижности) проводимость всей смеси при изменении количества ионов может как увеличиваться, так и уменьшаться. Объясняется такой эффект электронейтральностью раствора. Если в некоторую область раствора поместить ионы с большой подвижностью, то в силу электронейтральности всей смеси они будут вытеснены менее подвижными ионами, и в данной области общая проводимость раствора

Работа выполнена при поддержке Министерства образования и науки Российской Федерации (базовая часть технического задания № 1.5169.2017/8.9). 
понизится. Напротив, если добавить в раствор компонент с малым значением подвижности, то он вытеснит более подвижные соседние ионы (см. [5]). При построении математической модели указанные эффекты приводят к тому, что система квазилинейных дифференциальных уравнений первого порядка, описывающая процесс, может иметь как гиперболический тип, так и эллиптический, в зависимости от изменения концентраций отдельных компонент смеси.

В данной работе представлены исследования процесса переноса веществ в двухкомпонентной смеси (см. также [1-3,7]). Рассматриваются случаи гиперболических и эллиптических уравнений. Для анализа изменений с течением времени концентраций компонент смеси использовались пространственно-периодические начальные распределения. Решение задачи проводилось с помощью метода годографа и метода конечных объемов.

2. Постановка задачи. Процесс зонального электрофореза для двухкомпонентной смеси описывается системой двух квазилинейных уравнений следующего вида (см. [2, 5]):

$$
\boldsymbol{U}_{t}+A(\boldsymbol{U}) \boldsymbol{U}_{x}=0
$$

где

$$
\boldsymbol{U}=\left(\begin{array}{c}
u^{1} \\
u^{2}
\end{array}\right), \quad \boldsymbol{H}=\left(\begin{array}{c}
\frac{\mu^{1} u^{1}}{1+s} \\
\frac{\mu^{2} u^{2}}{1+s}
\end{array}\right), \quad A_{k}^{j}(\boldsymbol{U})=\frac{\partial \boldsymbol{H}^{j}(\boldsymbol{U})}{\partial u^{k}}, \quad j, k=1,2 .
$$

Здесь $\mu^{k} \equiv$ const и $u^{k}=u^{k}(x, t)(-\infty<x<\infty, t>0)$ - подвижности и «эффективные» концентрации отдельных компонент смеси, а $(1+s)=1+u^{1}+u^{2}-$ полная проводимость всей смеси в целом. Әффективные концентрации $u^{k}$ определяются через концентрации компонент смеси и их зарядности. Поэтому они могут принимать как положительные, так и отрицательные значения. Однако проводимость всей смеси $(1+s)$ величина всегда положительная. В начальный момент процесса разделения смеси известны концентрации каждой компоненты

$$
u^{k}(x, 0)=\tilde{u}^{k}(x), \quad k=1,2 .
$$

Решение задачи (1)-(2) во многом зависит от типа уравнений системы (1), который может быть как гиперболическим, так и эллиптическим (см. [2,3,7]). Как правило, дифференциальные уравнения в частных производных первого порядка вида (1) имеют гиперболический тип. Однако в тех случаях, когда концентрация компонент смеси велика и проводимость всей смеси в целом может уменьшаться при увеличении концентраций, тип квазилинейных уравнений изменяется на эллиптический.

Введем обозначение

$$
B=\mu^{1}+\mu^{2}+\mu^{2} u^{1}+\mu^{1} u^{2} .
$$

Тип уравнений (1) определяется значением величины

$$
D=B^{2}-4 \mu^{1} \mu^{2}(1+s) .
$$

Из соотношения (4) видно, что на $\left(u^{1}, u^{2}\right)$-плоскости линия $D=0$ является параболой (см. рис. 1$)$. Известно, что в области $D>0,1+s>0$ тип уравнений гиперболический, в области $D<0-$ эллиптический (см. [7]).

3. Методы решения. Рассмотрим два способа решения задачи (1)-(2). Первый из них - это метод годографа, основанный на законах сохранения (см. [11]). Известно, что система $n$ квазилинейных гиперболических уравнений интегрируема, если выполняется условие полугамильтоновости

$$
\partial_{i}\left(\frac{\partial_{j} \lambda^{k}}{\lambda^{j}-\lambda^{k}}\right)=\partial_{j}\left(\frac{\partial_{i} \lambda^{k}}{\lambda^{i}-\lambda^{k}}\right), \quad i \neq j \neq k, \quad \partial_{i}=\frac{\partial}{\partial R^{i}}
$$

где $R^{k}=R^{k}(x, t)$ - инварианты Римана, $\lambda^{k}=\lambda^{k}(R)$ - собственные значения матрицы системы $A_{j}^{i}(u), i, j, k=1,2, \ldots, n$. 


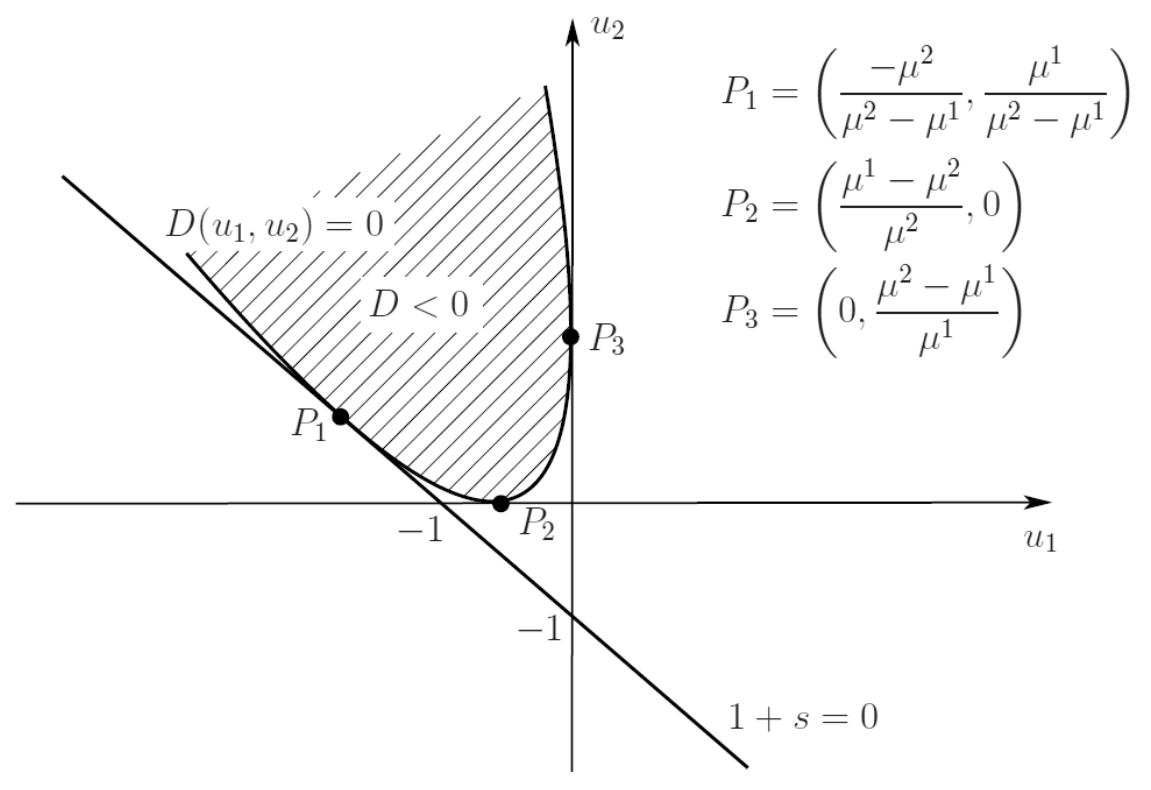

Рис. 1. Области гиперболичности и эллиптичности уравнения (1)

Так как система из двух уравнений гиперболического типа всегда приводится к инвариантам Римана $R^{1}, R^{2}$

$$
\frac{\partial R^{k}}{\partial t}+\lambda^{k}(R) \frac{\partial R^{k}}{\partial x}=0, \quad \lambda^{k}=R^{k} R^{1} R^{2}, \quad k=1,2,
$$

и условие полугамильтоновости будет выполняться автоматически, то система (1) разрешима, причем она может быть преобразована в однородную систему двух линейных гиперболических уравнений с коэффициентами, зависящими от $\left(u^{1}, u^{2}\right)$, относительно функций $t\left(u^{1}, u^{2}\right), x\left(u^{1}, u^{2}\right)$ (см. [8]). Такой обмен ролями зависимых и независимых переменных и есть обычный метод годографа.

С математической точки зрения для формального применения обычного метода годографа гиперболичность системы квазилинейных уравнений не является строго необходимым условием. Система (1) интегрируема и в случае комплексных собственных значений матрицы $\boldsymbol{A}$, т.е. метод годографа применим и для решения эллиптических квазилинейных уравнений.

Второй метод-метод конечных объемов (MKO). Это численный метод интегрирования систем дифференциальных уравнений в частных производных гиперболического типа, основанный на законах сохранения в каждом конечном (контрольном) объеме рассматриваемого пространства.

4. Метод годографа. Инварианты Римана в системе (5) вычисляются по формулам (см. [5])

$$
R^{1}=\frac{B-\sqrt{D}}{2(1+s)}, \quad R^{2}=\frac{B+\sqrt{D}}{2(1+s)}
$$

где $B, D$ определены соотношениями (3) и (4) соответственно.

Отметим, что эллиптичность уравнений (1) соответствует комплексно сопряженным собственным значениям $\lambda^{i}$ и инвариантам Римана $R^{i}$.

Связь между переменными уравнений (1) и (5) дается соотношениями

$$
u^{1}=\frac{\mu^{2}\left(R^{1}-\mu^{1}\right)\left(R^{2}-\mu^{1}\right)}{R^{1} R^{2}\left(\mu^{1}-\mu^{2}\right)}, \quad u^{2}=\frac{\mu^{1}\left(R^{1}-\mu^{2}\right)\left(R^{2}-\mu^{2}\right)}{R^{1} R^{2}\left(\mu^{1}-\mu^{2}\right)} .
$$


Удобно далее использовать замену переменных $R^{i}=1 / K^{i}, i=1,2$. Тогда в новых переменных, которые также являются инвариантами Римана, уравнения (5) примут вид

$$
K_{t}^{1}+\Lambda^{1} K_{x}^{1}=0, \quad K_{t}^{2}+\Lambda^{2} K_{x}^{2}=0,
$$

где

Для уравнений (8) на некотором контуре

$$
\Lambda^{1}=\frac{1}{K^{1} K^{1} K^{2}}, \quad \Lambda^{2}=\frac{1}{K^{2} K^{1} K^{2}} .
$$

$$
\Gamma=\{(x, t): x=x(\tau), t=t(\tau), a \leqslant \tau \leqslant b\},
$$

не являющемся характеристикой, определены начальные данные

$$
\left.K^{1}\right|_{\Gamma}=K_{0}^{1}(\tau),\left.\quad K^{2}\right|_{\Gamma}=K_{0}^{2}(\tau) .
$$

Здесь $K_{0}^{i}(\tau)(i=1,2)$ - функции, определяемые начальными распределениями (2).

В [11] описан метод годографа, в котором замена $\left(K^{1}, K^{2}\right) \leftrightarrows(x, t)$ позволяет получить неявные решения $t=t\left(K^{1}, K^{2}\right)$ и $x=x\left(K^{1}, K^{2}\right)$ уравнений $(8)$, позволяющие восстановить явный вид неизвестных функций $u^{1}(x, t), u^{2}(x, t)$ при помощи соотношений $(7)$. Следуя алгоритму, описанному в [11], предположим, что для (8) имеется закон сохранения

$$
\varphi_{t}+\psi_{x}=0
$$

где $\varphi\left(K^{1}, K^{2}\right)$ - плотность, $\psi\left(K^{1}, K^{2}\right)$ - плотность потока, для которых выполнены уравнения

$$
\psi_{K^{1}}=\Lambda^{1} \varphi_{K^{1}}, \quad \psi_{K^{2}}=\Lambda^{2} \varphi_{K^{2}} .
$$

Условия разрешимости приводят к линейным дифференциальным уравнениям второго порядка с переменными коэффициентами:

$$
\begin{gathered}
\varphi_{K^{1} K^{2}}-\frac{1}{K^{2}-K^{1}} \varphi_{K^{1}}+\frac{1}{K^{2}-K^{1}} \varphi_{K^{2}}=0, \\
\psi_{K^{1} K^{2}}+\frac{K^{1}}{K^{2}\left(K^{1}-K^{2}\right)} \psi_{K^{1}}-\frac{K^{2}}{K^{1}\left(K^{1}-K^{2}\right)} \psi_{K^{2}}=0 .
\end{gathered}
$$

Для определения функций $\varphi\left(K^{1}, K^{2}\right), \psi\left(K^{1}, K^{2}\right)$ эти уравнения дополняются условиями

$$
\begin{aligned}
& \left.\left(\psi-\Lambda^{1} \varphi\right)\right|_{K^{1}=k^{1}}=1,\left.\quad\left(\psi-\Lambda^{2} \varphi\right)\right|_{K^{2}=k^{2}}=-1 ; \\
& \left.\left(\psi-\Lambda^{1} \varphi\right)\right|_{K^{1}=k^{1}}=\left.\Lambda^{1}\right|_{K^{1}=k^{1}},\left.\quad\left(\psi-\Lambda^{2} \varphi\right)\right|_{K^{2}=k^{2}}=-\left.\Lambda^{2}\right|_{K^{2}=k^{2}} .
\end{aligned}
$$

Решением $\varphi\left(K^{1}, K^{2}\right)$ задачи $(12),(14)$ с точностью до множителей является функция РиманаГрина $\Phi\left(K^{1}, K^{2} \mid k^{1}, k^{2}\right)$ :

$$
\varphi^{t}\left(K^{1}, K^{2}\right)=\frac{2}{\Lambda^{2}\left(k^{1}, k^{2}\right)-\Lambda^{1}\left(k^{1}, k^{2}\right)} \Phi\left(K^{1}, K^{2} \mid k^{1}, k^{2}\right) .
$$

Тогда, согласно условиям (11), получаем

$$
\psi^{t}\left(k^{1}, k^{2}\right)=\frac{\Lambda^{1}\left(k^{1}, k^{2}\right)+\Lambda^{2}\left(k^{1}, k^{2}\right)}{2} \varphi^{t}\left(k^{1}, k^{2}\right),
$$

где $K^{1}=k^{1}, K^{2}=k^{2}$.

Для исследуемых уравнений функция Римана-Грина имеет вид (см. [9])

$$
\Phi\left(K^{1}, K^{2} \mid k^{1}, k^{2}\right)=\frac{\left(K^{1}+K^{2}\right)\left(k^{1}+k^{2}\right)-2 K^{1} K^{2}-2 k^{1} k^{2}}{\left(k^{1}-k^{2}\right)^{2}} .
$$

Плотность потока $\psi\left(K^{1}, K^{2}\right)$ определяется из задачи (13), (15)

$$
\psi^{x}\left(K^{1}, K^{2}\right)=\frac{2 \Lambda^{1}\left(k^{1}, k^{2}\right) \Lambda^{2}\left(k^{1}, k^{2}\right)}{\Lambda^{2}\left(k^{1}, k^{2}\right)-\Lambda^{1}\left(k^{1}, k^{2}\right)} \Phi\left(K^{1}, K^{2} \mid k^{1}, k^{2}\right) .
$$

Для определения функции $\varphi^{x}$ интегрируется одно из уравнений в (11):

$$
\varphi^{x}\left(k^{1}, K^{2}\right)=\frac{\psi^{x}\left(k^{1}, K^{2}\right)}{\Lambda^{1}\left(k^{1}, K^{2}\right)}-1 \quad \text { или } \quad \varphi^{x}\left(K^{1}, k^{2}\right)=\frac{\psi^{x}\left(K^{1}, k^{2}\right)}{\Lambda^{2}\left(K^{1}, k^{2}\right)}+1 .
$$


Рассмотрим случай, когда контур $\Gamma$, определенный $(9)$, является отрезком оси $t=t_{0}$. Тогда начальные условия (10) запишутся в виде

$$
\left.K^{1}\right|_{t=t_{0}}=K_{0}^{1}(\tau),\left.\quad K^{2}\right|_{t=t_{0}}=K_{0}^{2}(\tau), \quad a \leqslant \tau \leqslant b .
$$

Отсюда следует, что

$$
k^{1}=K_{0}^{1}(b), \quad k^{2}=K_{0}^{2}(a) .
$$

Неявное решение задачи Коши (8), (10) имеет вид

$$
t=t_{0}+\frac{1}{2} \int_{a}^{b} \varphi^{t} d \tau, \quad x=\frac{a+b}{2}+\frac{1}{2} \int_{a}^{b} \varphi^{x} d \tau .
$$

Таким образом, выбор естественного начального условия при $t=t_{0}$, а не на произвольном контуре $\Gamma$, упрощает формулы, в частности, отпадает необходимость в определении функции $\psi^{t}$.

Рассмотрим решение задачи задачи Коши (8), (10) в случае гиперболичности уравнений (8). Тогда собственные значения $\lambda^{k}$ и инварианты Римана $R^{k}$ будут вещественными, а линии уровня $t(a, b), x(a, b)$, рассчитанные по формулам (18), описываются соотношениями

$$
\begin{aligned}
t(a, b) & =t_{0}+\frac{\left(k^{1} k^{2}\right)^{2}\left(-2 k^{1} k^{2}(b-a)+\left(k^{1}+k^{2}\right) F-2 G\right)}{\left(k^{1}-k^{2}\right)^{3}}, \\
x(a, b) & =\frac{a+b}{2}+\frac{\left(\left(k^{1}\right)^{2}-4 k^{1} k^{2}+\left(k^{2}\right)^{2}\right)\left(k^{1}+k^{2}\right)(b-a)}{2\left(k^{1}-k^{2}\right)^{3}}+\frac{2 k^{1} k^{2} F-\left(k^{1}+k^{2}\right) G}{\left(k^{1}-k^{2}\right)^{3}},
\end{aligned}
$$

где $k^{1}=K_{0}^{1}(b), k^{2}=K_{0}^{2}(a)$,

$$
F=\int_{a}^{b}\left(K_{0}^{1}(\tau)+K_{0}^{2}(\tau)\right) d \tau, \quad G=\int_{a}^{b} K_{0}^{1}(\tau) K_{0}^{2}(\tau) d \tau .
$$

Для восстановления явного решения выбираем произвольную точку $\left(a_{*}, b_{*}\right)$ и для некоторого параметра $\rho$ на изолинии $t_{*}=t\left(a_{*}, b_{*}\right)$ решаем задачу Коши для обыкновенных дифференциальных уравнений (см. [7, гл. 3])

$$
\frac{d a}{d \rho}=-t_{b}(a, b), \quad \frac{d b}{d \rho}=\left.t_{a}(a, b) a\right|_{\rho=0}=a_{*},\left.\quad b\right|_{\rho=0}=b_{*} .
$$

В случае, когда инварианты Римана $K^{1}, K^{2}$ комплексно сопряжены, ключевым моментом в построении решения поставленной задачи (8), (10) будет требование комплексной сопряженности параметров $a$ и $b$ в (9).

Введем следующие обозначения:

$$
\begin{gathered}
\boldsymbol{a}=u+i v, \quad \boldsymbol{b}=u-i v, \quad \boldsymbol{a}^{*}=\boldsymbol{b}, \\
\boldsymbol{K}=K^{1}=P+i Q, \quad \boldsymbol{K}^{*}=K^{2}=P-i Q, \\
\boldsymbol{k}=k^{1}=p+i q, \quad \boldsymbol{k}^{*}=k^{2}=p-i q,
\end{gathered}
$$

где $P=P(u, v), Q=Q(u, v), p=p(u, v), q=q(u, v)$ - вещественные функции от вещественных переменных $u, v$.

Таким образом, задачу (8), (10) можно переписать в комплексной форме:

$$
\boldsymbol{K}|\boldsymbol{K}|^{2} \boldsymbol{K}_{t}+\boldsymbol{K}_{x}=0,\left.\quad \boldsymbol{K}\right|_{t=t_{0}}=\boldsymbol{K}_{0}(\tau) .
$$

Здесь $\boldsymbol{K}_{0}(\tau)=P_{0}(\tau)+i Q_{0}(\tau)$ - известная функция.

Соотношения (18) с учетом введенных замен и равенств (17) и (20) запишутся следующим образом:

$$
\begin{aligned}
t(u, v) & =t_{0}+\frac{\left(p^{2}+q^{2}\right)^{2}\left(4 i v\left(p^{2}+q^{2}\right)+2 p F-2 G\right)}{(2 i q)^{3}} \\
x(u, v) & =u+\frac{2\left(p^{2}+q^{2}\right) F-2 p G-2 i p v\left(4 p^{2}-6\left(p^{2}+q^{2}\right)\right)}{(2 i q)^{3}}
\end{aligned}
$$


где

$$
F(u, v)=2 \int_{u+i v}^{u-i v} P_{0}(\tau) d \tau, \quad G(u, v)=\int_{u+i v}^{u-i v}\left(P_{0}^{2}(\tau)+Q_{0}^{2}(\tau)\right) d \tau
$$

Легко показать, что функции $F(u, v)$ и $G(u, v)$ чисто мнимые. Положим

$$
F^{0}(u, v)=\operatorname{Im} F(u, v), \quad G^{0}(u, v)=\operatorname{Im} G(u, v) .
$$

Очевидно, что функции $F^{0}, G^{0}$ - вещественные функции, зависящие от вещественных переменных $u, v$.

Используя (20), из начального условия получим соотношения для функций $p$ и $q$ :

$$
p(u, v)=\operatorname{Re} \boldsymbol{K}_{0}(b), \quad q(u, v)=\operatorname{Im} \boldsymbol{K}_{0}(b) .
$$

Таким образом, получаем неявное решение задачи Коши (21):

$$
\begin{aligned}
& t(u, v)=t_{0}-\frac{\left(p^{2}+q^{2}\right)^{2}\left(p F_{0}-G_{0}+2 v\left(p^{2}+q^{2}\right)\right)}{4 q^{3}}, \\
& x(u, v)=u-\frac{p G^{0}-\left(p^{2}+q^{2}\right) F_{0}-2 p\left(p^{2}+3 q^{2}\right)}{4 q^{3}},
\end{aligned}
$$

которое полностью определено начальными условиями.

Для восстановления явного решение задачи Коши на некоторых линиях уровня неявного решения, отвечающих фиксированным значениям $t_{*}=$ const, решаем задачу Коши

$$
\begin{array}{ll}
\frac{d u}{d \rho}=-t_{v}(u, v) q^{4}(u, v), & \frac{d v}{d \rho}=t_{u}(u, v) q^{4}(u, v), \\
\left.u\right|_{\rho=0}=u_{*}, & \left.v\right|_{\rho=0}=v_{*}
\end{array}
$$

(см. [7, гл. 7]), где $\rho$ - некоторый параметр, а значения величин $u_{*}, v_{*}$ идентифицируют изохрону $t_{*}=t\left(u_{*}, v_{*}\right)$.

Производные $t_{u}, t_{v}$ вычисляются с помощью (22), а пространственная координата на изохроне $x=x(u(\rho), v(\rho))-\mathrm{c}$ помощью $(23)$.

Правая часть дифференциальных уравнений (24) определена с точностью до произвольного множителя. Использованный здесь множитель $q^{4}(u, v)$ позволяет избавиться от особенности правых частей в точке $q=0$.

Определив значения $u(\rho), v(\rho)$ на изохроне $t=t_{*}\left(u_{*}, v_{*}\right)$, найдем функции $p(u, v), q(u, v)$. Далее вычислим концентрации компонент смеси, которые связаны с инвариантами Римана при помощи соотношений (7):

$$
u^{1}=\frac{\mu^{2}\left(1-2 \mu^{1} p+\mu^{1} \mu^{1}\left(p^{2}+q^{2}\right)\right)}{\mu^{1}-\mu^{2}}, \quad u^{2}=\frac{\mu^{1}\left(1-2 \mu^{2} p+\mu^{2} \mu^{2}\left(p^{2}+q^{2}\right)\right)}{\mu^{2}-\mu^{1}} .
$$

5. Метод конечных объемов. Пусть $D>0,1+s>0$. Найдем решение поставленной задачи с помощью метода конечных объемов (МКО; см. [6]).

В исходной постановке задача Коши (1)-(2) определена на бесконечной оси $-\infty<x<\infty$. Однако для численной реализации удобно выбрать такой конечный интервал $[a, b]$, на котором происходят основные изменения концентраций. Выбранный промежуток разбивается на ячейки

$$
\Omega_{i}=\left[x_{i-1 / 2}, x_{i+1 / 2}\right],
$$

которые имеют центры $x_{i}$. Используем обычную сетку с постоянным шагом $h=(b-a) / n$, т.е. $x_{i}=i h, i=0,1, \ldots, n$.

После интегрирования уравнения (1) по области каждой ячейки $\Omega_{i}$ имеем

$$
h \frac{d \boldsymbol{U}_{i}}{d t}=\boldsymbol{H}_{i+1 / 2}-\boldsymbol{H}_{i-1 / 2},
$$

где $\boldsymbol{U}_{i}=\left(u^{1}\left(x_{i}, t\right), u^{2}\left(x_{i}, t\right)\right)^{T}$. 

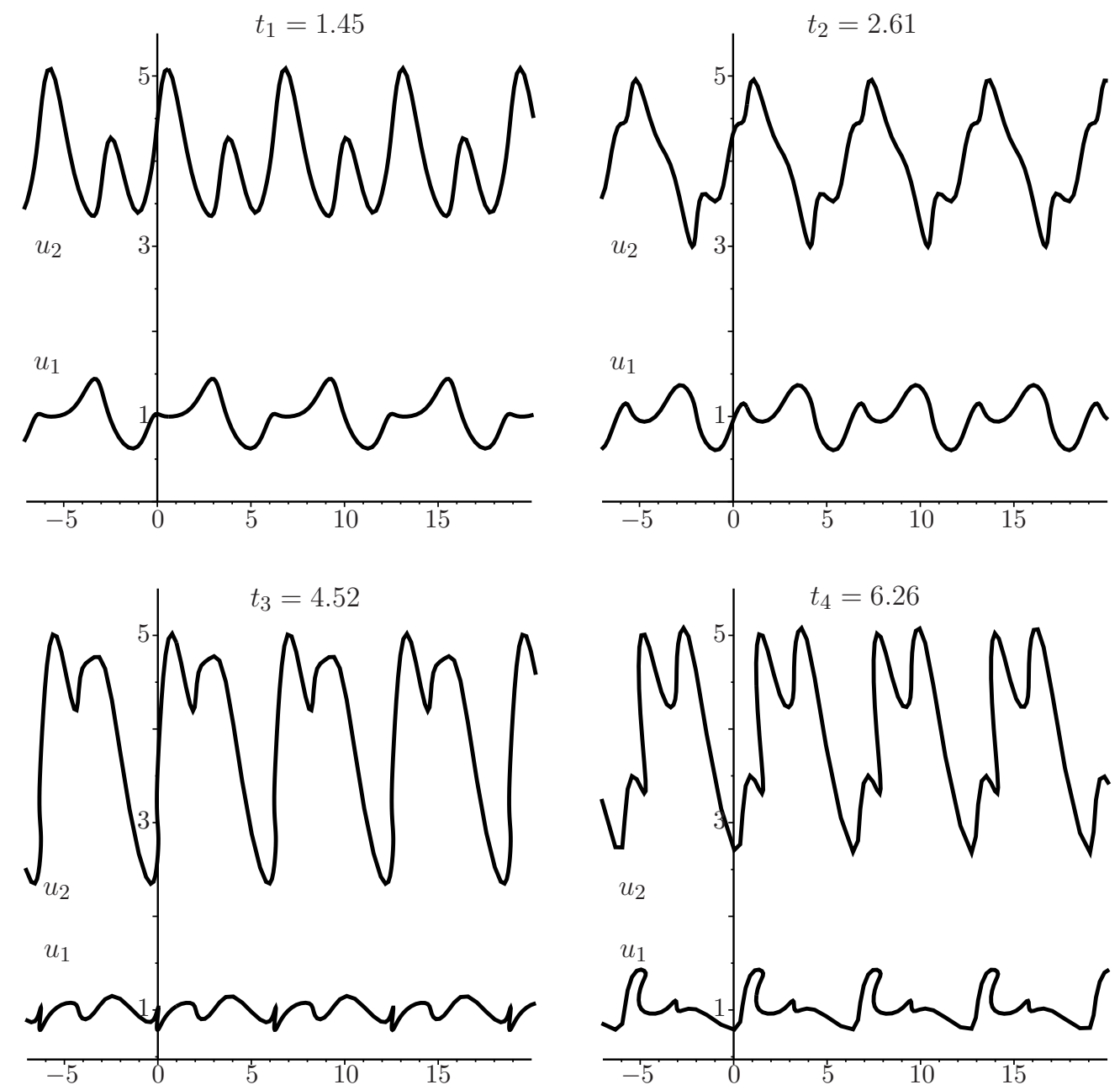

Рис. 2. Концентрации компонент смеси $u_{1}(x, t), u_{2}(x, t)$, вычисленные с помощью метода годографа для начальных условий (27), в моменты времени $t=1,45,2,61,4,52,6,26$ при $a_{*}=-0,29$, $-1,13,-2,35,-3,67, b_{*}=0,40$ соответственно.

Если применить временну́ю аппроксимацию, как в явном методе Эйлера, то получим вместо уравнений в частных производных (1) систему обыкновенных дифференциальных уравнений с начальными условиями (2):

$$
\frac{\boldsymbol{U}_{i}^{m+1}-\boldsymbol{U}_{i}^{m}}{\tau}=\frac{\boldsymbol{H}_{i+1 / 2}^{m}-\boldsymbol{H}_{i-1 / 2}^{m}}{h} .
$$

Здесь верхний индекс $m$ означает величины $\boldsymbol{U}_{i}$ и $\boldsymbol{H}_{i}$ в момент времени $t_{m}$. Отметим, что $t_{m+1}=$ $t_{m}+\tau$, где $\tau$-шаг по времени.

Для аппроксимации значений вектора $\boldsymbol{H}$ на границах отрезка $\Omega_{i}$ используем HLL-аппроксимацию (см. [10]).

Вычислим собственные значения матрицы $A(\boldsymbol{U})$ :

$$
\lambda_{1,2}(\boldsymbol{U})=\frac{B \mp \sqrt{D}}{2(1+s)^{2}},
$$



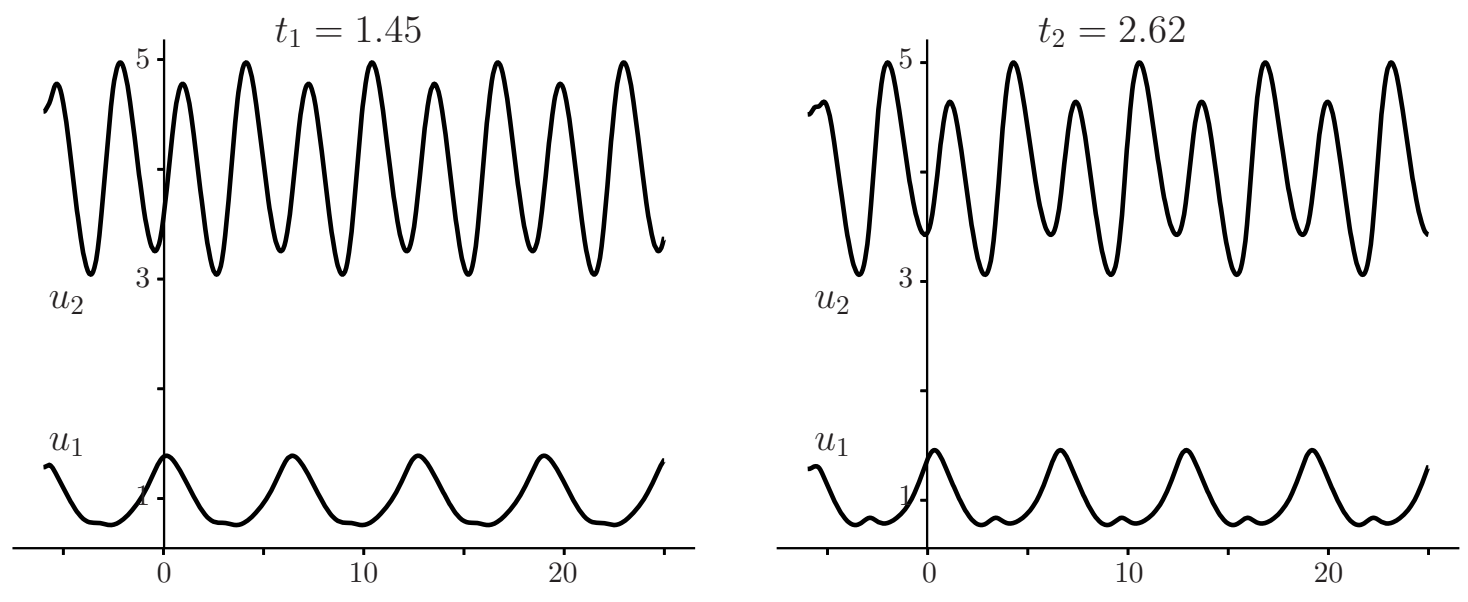

$$
t_{3}=4.52
$$
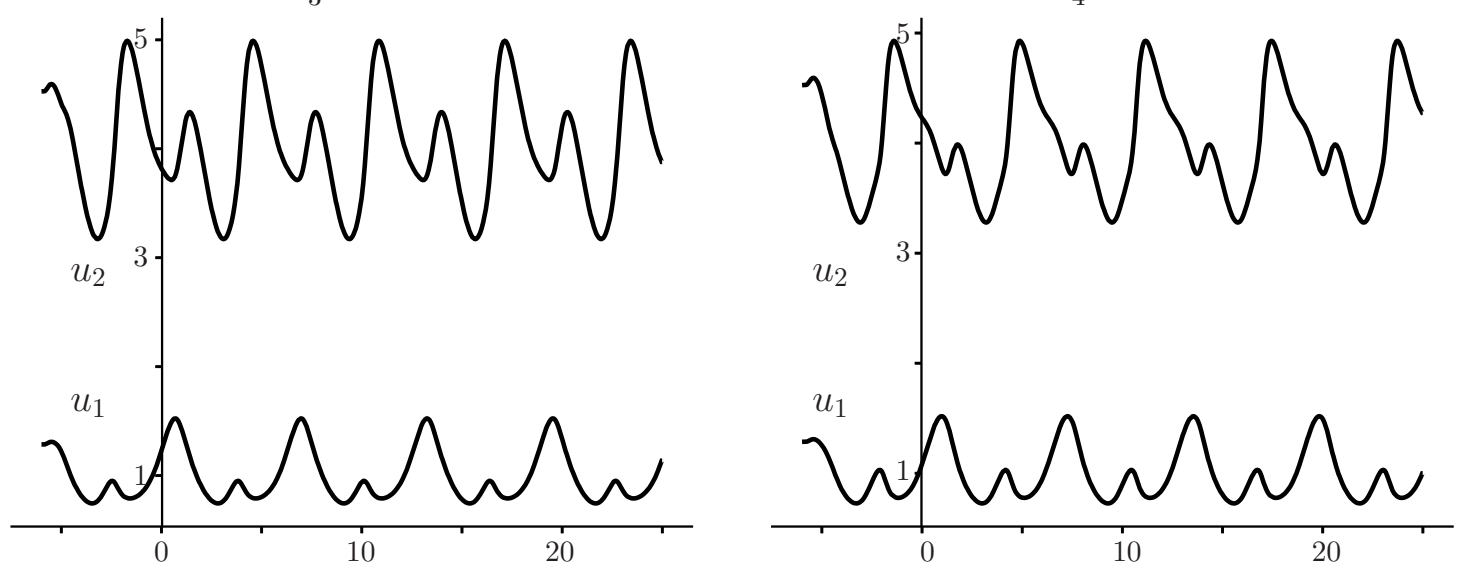

Рис. 3. Концентрации компонент смеси $u_{1}(x, t), u_{2}(x, t)$, вычисленные с помощью МКО для начальных условий (27), в моменты времени $t=1,45,2,61,4,52,6,26$.

где $B$ и $D$ определяются соотношениями (3) и (4) соответственно. Далее для границ ячейки $\Omega_{i}$ определим численные потоки

$$
\begin{gathered}
\mathcal{H}(\boldsymbol{a}, \boldsymbol{b})= \begin{cases}\boldsymbol{H}(\boldsymbol{a}), & \lambda_{L}>0, \\
\boldsymbol{H}(\boldsymbol{b}), & \lambda_{R}<0, \\
\frac{\lambda_{R} \boldsymbol{H}(\boldsymbol{a})-\lambda_{L} \boldsymbol{H}(\boldsymbol{b})+\lambda_{L} \lambda_{R}(\boldsymbol{b}-\boldsymbol{a})}{\lambda_{R}-\lambda_{L}}, & \lambda_{L} \leqslant 0 \leqslant \lambda_{R},\end{cases} \\
\lambda_{L}=\min \left\{\lambda_{1}(\boldsymbol{a}), \lambda_{1}(\boldsymbol{b}), \lambda_{2}(\boldsymbol{a}), \lambda_{2}(\boldsymbol{b})\right\}, \quad \lambda_{R}=\max \left\{\lambda_{1}(\boldsymbol{a}), \lambda_{1}(\boldsymbol{b}), \lambda_{2}(\boldsymbol{a}), \lambda_{2}(\boldsymbol{b})\right\} .
\end{gathered}
$$

Таким образом, разностная схема (25) окончательно примет вид

$$
\boldsymbol{U}_{i}^{m+1}=\frac{\tau}{h}\left[\mathcal{H}\left(\boldsymbol{U}_{i-1}^{m}, \boldsymbol{U}_{i}^{m}\right)-\mathcal{H}\left(\boldsymbol{U}_{i}^{m}, \boldsymbol{U}_{i+1}^{m}\right)\right]+\boldsymbol{U}_{i}^{m}, \quad m=1,2, \ldots,
$$

где $\boldsymbol{U}_{i}^{m}=\left(u^{1}\left(x_{i}, t_{m}\right), u^{2}\left(x_{i}, t_{m}\right)\right)^{T}, \boldsymbol{U}_{i}^{0}=\left(\tilde{u}^{1}\left(x_{i}\right), \tilde{u}^{2}\left(x_{i}\right)\right)^{T}, i=0,1, \ldots, n$.

6. Примеры решений задач Коши. В случае квазилинейных уравнений в частных производных первого порядка независимые переменные $t$ и $x$ в некотором смысле равноправны. Поэтому о задаче с данными на какой-либо линии (9) принято говорить как о задаче Коши (см. [8]). Для эллиптических уравнений вовсе не принято говорить о начальных данных, однако далее используем термин задача Коши, так как концентрации заданы на отрезке оси $t=t_{0}$. 

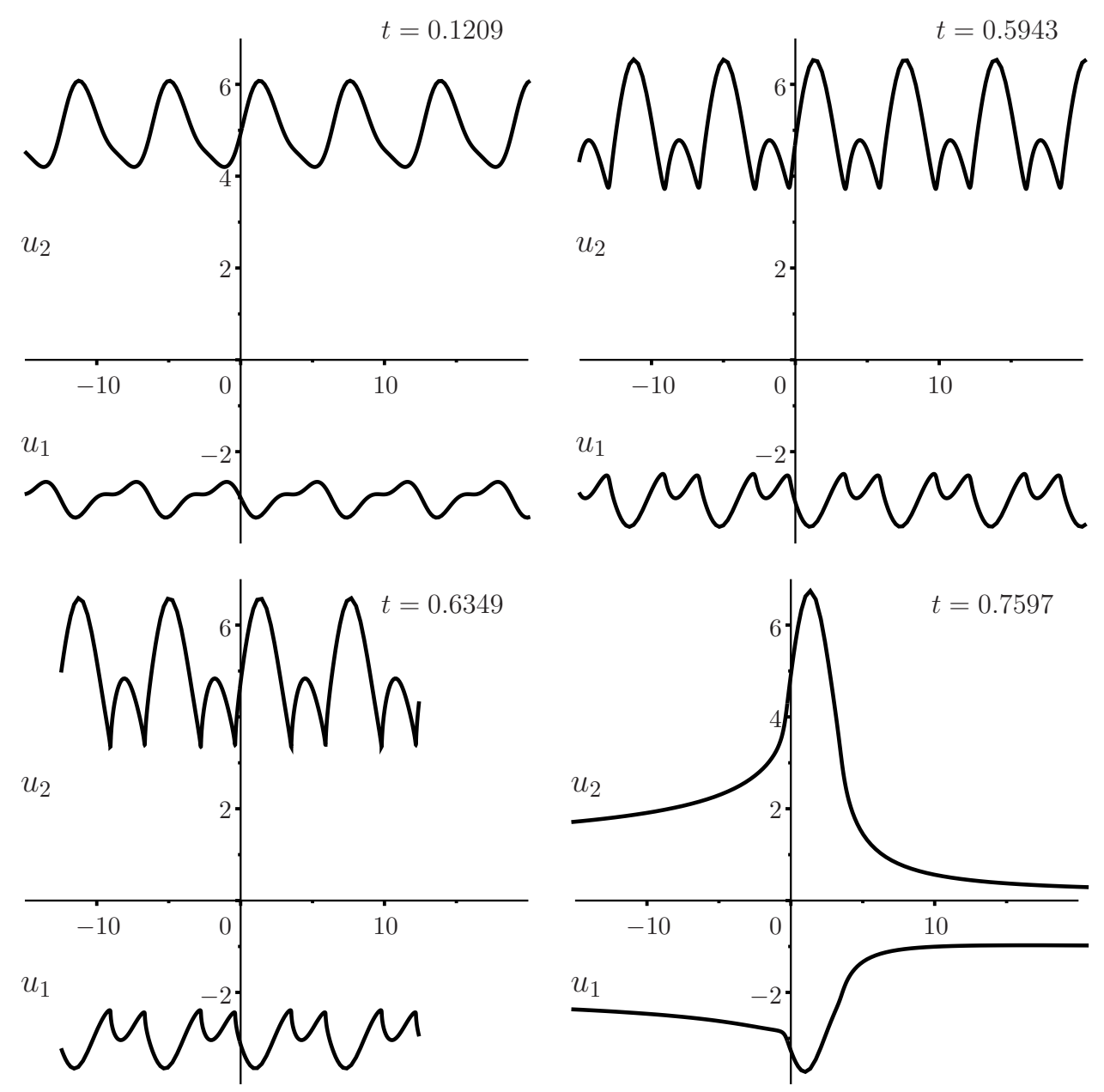

Рис. 4. Концентрации компонент смеси $u_{1}(x, t), u_{2}(x, t)$, вычисленные с помощью метода годографа для начальных условий (28), в моменты времени $t=0,1209,0,5943,0,6348,0,7597$.

Рассмотрим случай гиперболичности уравнений (1). Пусть начальные распределения компонент смеси (2) заданы периодическим по пространству условием

$$
\tilde{u}^{1}(x)=\alpha^{1}+\beta^{1} \cos \omega^{1} x, \quad \tilde{u}^{2}(x)=\alpha^{2}+\beta^{2} \sin \omega^{2} x,
$$

где $\alpha^{k}, \beta^{k}, \gamma^{k}, \omega^{k}(k=1,2)$ - некоторые константы. Положим

$$
\mu^{1}=1, \quad \mu^{2}=3, \quad \alpha^{1}=1, \quad \alpha^{2}=4, \quad \beta^{1}=0,3, \quad \beta^{2}=0,9, \quad \omega^{1}=1, \quad \omega^{2}=2 .
$$

Для этих параметров решим задачу Коши (1)-(2) с начальными распределениями (27) методом годографа и методом конечных объемов. Графики изменения концентраций $u_{1}(x, t)$ и $u_{2}(x, t)$ в одинаковые моменты времени показаны на рис. 2 и 3.

Известно, что сплошные среды, которые описываются уравнениями эллиптического типа, ведут себя как неустойчивые квазигазовые среды типа газа Чаплыгина. Для исследования таких сред часто используются пространственно-периодические начальные данные. Рассмотрим такие же начальные распределения концентраций и для анализа эволюции решений задачи зонального электрофореза (21) (см. также $[2,3,7])$. Пусть $K_{0}^{1}(\tau)=P_{0}+i Q_{0}, K_{0}^{2}(\tau)=P_{0}-i Q_{0}$, где

$$
P_{0}(\tau)=-\varepsilon \sin \tau \cos \tau, \quad Q_{0}(\tau)=1+\varepsilon \sin \tau .
$$

Здесь $\varepsilon$ - амплитуда возмущения состояния равновесия $P_{0}=0, Q_{0}=1$, которое может возникнуть, например, из-за нестабильности внешнего электрического поля.

На рис. 4 представлены результаты расчетов при $\mu^{1}=1, \mu^{2}=3, \varepsilon=0,1$. 
7. Заключение. Решение задачи электрофореза для двухкомпонентной смеси методом годографа на основе закона сохранения можно использовать для любых, в том числе и не гладких, начальных данных. Фактически этот метод позволяет сводить решение задачи Коши для двух квазилинейных уравнений в частных производных первого порядка к решению некоторой задачи Коши для обыкновенных дифференциальных уравнений. Более того, метод позволяет получить точное решение, хотя и в неявном виде, в аналитической форме, в частности, построить уравнения для линий уровня решения. Решение задачи Коши для обыкновенных дифференциальных уравнений требуется лишь для преобразования неявного решения в явное. В этом смысле метод является полуаналитическим, и его точность зависит лишь от точности интегрирования системы обыкновенных дифференциальных уравнений. Иными словами, не требуется никаких аппроксимаций уравнений в частных производных для построения вычислительных схем. Конечно, метод построения аналитического решения применим лишь тогда, когда для линейного уравнения в частных производных второго порядка (в статье это уравнение (12)) удается указать функцию Римана-Грина. Однако набор уравнений, имеющих известные функции Римана-Грина, достаточно широк (см., например, [4,7,9,11]).

Приведенные численные решения методом конечных объемов показывают, что в случае гиперболических уравнений результаты расчетов мало отличаются от решений, полученных методом годографа, хотя на больших временах совпадение результатов будет не таким хорошим. Для уравнений эллиптического типа метод конечных объемов в стандартном виде не пригоден. В частности, следует существенно изменять схему построения численных потоков, и возможность использования HLL-аппроксимации нуждается в детальном исследовании. Напротив, метод годографа и в случае уравнений эллиптического типа позволяет эффективно строить решения.

\section{СПИСОК ЛИТЕРАТУРЫ}

1. Долгих Т. Ф. Метод конечных объемов для решения задачи зонального электрофореза// XIX Междунар. конф. «Современные проблемы механики сплошной среды» (Ростов-на-Дону, 15-18 октября 2018), 2018. - C. 94-98.

2. Долгих T. Ф. Решение задачи о переносе массы под действием электрического поля в двухкомпонентной смеси// Изв. Сев-Кавказ. науч. центра высш. школы. Естеств. науки. - 2017. - № 3-1 (195-1). C. $28-35$.

3. Долгих Т. Ф., Жуков М. Ю., Ширяева Е. В. Решение эллиптических уравнений с периодическими данными для задачи зонального электрофореза// Вестн. Воронеж. гос. ун-та. Сер. Физ. Мат. - 2017. - № 2 . - С. $85-96$.

4. Жданов С. К., Трубников Б. А. Квазигазовые неустойчивые среды. - М.: Наука, 1971.

5. Жуков М. Ю. Массоперенос электрическим полем. - Ростов-на-Дону: РГУ, 2005.

6. Жуков М. Ю., Ширяева Е. В. Математическое моделирование процесса седиментации примеси в потоке жидкости. - Ростов-на-Дону: ЮФУ, 2016.

7. Жуков М. Ю., Ширяева Е. В., Долгих Т. Ф. Метод годографа для решения гиперболических и эллиптических квазилинейных уравнений. - Ростов-на-Дону: ЮФУ, 2015.

8. Рождественский Б. Л., Яненко Н. Н. Системы квазилинейных уравнений. - М.: Наука, 1978.

9. Copson E. T. On the Riemann-Green Function// Arch. Rat. Mech. Anal. - 1958. - 1. - P. 324-348.

10. Harten A. High resolution schemes for hyporbolic conservation laws// J. Comput. Phys. — 1997. — 135. - P. 260-278.

11. Senashov S. I., Yakhno A. Conservation laws, hodograph transformation and boundary value problems of plane plasticity// SIGMA. - 2012. - 8. - 071.

Долгих Татьяна Федоровна

Южный федеральный университет, Ростов-на-Дону

E-mail: dolgikh@sfedu.ru 